\title{
SEQUENTIAL CONDITIONS AND FREE TOPOLOGICAL GROUPS
}

\author{
EDWARD T. ORDMAN AND BARBARA V. SMITH-THOMAS
}

\begin{abstract}
Most of the results in this paper concern relationships between sequential properties of a pointed topological space $(X, p)$ and sequential properties of the Graev free topological group on $X$. In particular, it is shown that the free group over a sequential $k_{\omega}$-space is sequential, and that a nondiscrete sequential free group has sequential order equal to $\omega_{1}$ (the first uncountable ordinal). The free topological group on a space $X$ which includes a convergent sequence contains a closed subspace homeomorphic to $S_{\omega}$, a previously studied homogeneous, zero-dimensional sequential space. Finally, it is shown that there is no topological group homeomorphic to $S_{\omega}$.
\end{abstract}

0. Introduction. In this paper we discuss relationships between sequential properties of a pointed topological space $(X, p)$ and sequential properties of its Graev free topological group $F_{G}(X, p)$. Sequential spaces have been discussed in [D], [Fr ], [Fr $\mathbf{F}_{2}$ ], [A-Fr], [R]; we make heavy use of the space $S_{\omega}$ of sequential order $\omega_{1}$ constructed in [A-Fr]. The Graev free topological group $F_{G}(X, p)$ on a pointed Tychonoff space $(X, p)$ was introduced in $\left[\mathbf{G r}_{1}\right]$; its topology has proved to be rather intractable, but in the last few years good results have been obtained in the case when $X$ is a $k_{\omega}$-space, that is, a weak union of countably many compact subsets [01], [H-M], [MMO].

Definitions and preliminary results about sequential spaces appear in $\S 1 ; \S 2$ contains preliminaries about free topological groups and about $k_{\omega}$-spaces. $\$ 3$ contains results about sequential properties of free topological groups and their consequences. The result that $S_{\omega}$ supports no group structure (answering in the negative a question of S. P. Franklin) appears in $\$ 4$.

We thank S. P. Franklin for posing to us the question just mentioned, and for several helpful conversations and suggestions.

1. Sequential spaces. Our definition of sequential spaces, sequential order, and the particular example $S_{\omega}$, are based on [A-Fr].

A subset $U$ of a topological space $X$ is sequentially open if each sequence converging to a point in $U$ is eventually in $U$. The space $X$ is sequential if each sequentially open subset of $X$ is open. For each subset $A$ of $X$, let $s(A)$ denote the set of all limits of sequences of points of $A . X$ is of sequential order 1 ( $X$ is also called a Fréchet space) if $s(A)$ is the closure of $A$ for every $A$.

Presented to the Society, January 27, 1977; received by the editors February 5, 1979 and, in revised form, June 4, 1979.

AMS (MOS) subject classifications (1970). Primary 54D55, 22A99, $20 \mathrm{E} 05$.

Key words and phrases. Sequential space, sequential order, $k_{\omega}$-space, free topological group, Graev free topological group, sequential coreflection. 
We define higher sequential orders by induction. Let $s_{0}(A)=A$, and for each ordinal $\alpha=\beta+1$, let $s_{\alpha}(A)=s\left(s_{\beta}(A)\right)$. If $\alpha$ is a limit ordinal, let $s_{\alpha}(A)=$ $\cup\left\{s_{\beta}(A) \mid \beta<\alpha\right\}$. The sequential order of a sequential space $X$ is the least ordinal $\alpha$ such that $s_{\alpha}(A)$ is the closure of $A$ for every subset $A$ of $X$; this order always exists and does not exceed the first uncountable ordinal $\omega_{1}$.

By $\left(S_{1}, s_{0}\right)$ we mean a space consisting of a single convergent sequence $s_{1}, s_{2}, s_{3}, \ldots$, together with its limit point $s_{0}$ taken as the basepoint.

$\left(S_{2}, s_{0}\right)$ is a space obtained from $S_{1}$ by attaching to each isolated point $s_{n}$ of $S_{1}$ a sequence $s_{n, 1}, s_{n, 2}, s_{n, 3}, \ldots$ converging to $s_{n}$. $S_{2}$ may be viewed as a quotient of a disjoint union of convergent sequences; we give it the quotient topology. Inductively, we obtain the space $\left(S_{n+1}, s_{0}\right)$ from $\left(S_{n}, s_{0}\right)$ by attaching a convergent sequence to each isolated point of $\left(S_{n}, s_{0}\right)$ and giving the resulting set the quotient topology.

Finally, let $\left(S_{\omega}, s_{0}\right)$ be the union of the sets $S_{1} \subset S_{2} \subset S_{3} \subset \ldots$ with the weak union topology (a subset of $S_{\omega}$ is closed if and only if its intersection with each $S_{n}$ is closed in the topology of $S_{n}$ ).

We shall use heavily the following facts from $\left[\mathbf{F r}_{1}\right],\left[\mathrm{Fr}_{2}\right]$, and $[\mathrm{A}-\mathrm{Fr}]: S_{n}$ is sequential of order $n ; S_{\omega}$ is sequential of order $\omega_{1}$ and is countable, homogeneous, and zero-dimensional. A closed subspace of a sequential space (of order $\alpha$ ) is sequential (of order $\leqslant \alpha$ ). A quotient of a sequential space is sequential. The pointed union (one-point union) of sequential spaces is sequential. The weak union of a nest of sequential spaces is sequential.

By a theorem of Boehme [B], the cartesian product of a sequential space and a locally compact sequential space is again sequential. The local compactness condition cannot be entirely removed: the cartesian product of two sequential spaces (even of a metric space and a sequential space) need not be sequential. Let $(2, p)$ denote the rationals with basepoint $p=0$; let $(W, q)$ denote the union of countably many copies of the unit interval $[0,1]$ with all the basepoints $q=0$ identified and with the quotient topology. Then 2 is a sequential (in fact, a metric) space and $W$ is sequential, but $(2 \times W,(p, q))$ is not sequential. To see this think of $2 \times W$ as an infinite book: each leaf is $\mathcal{2} \times I$ and the spine is $2 \times\{q\}$. Pick a decreasing sequence of irrationals converging (in R) to 0 , say $\alpha_{1}, \alpha_{2}, \ldots$; in the $n$th leaf of the book pick a sequence $\left\{x_{n, i}\right\}_{i=1}^{\infty}$ in $2 \times(0,1]$ which converges to $\left(\alpha_{n}, 0\right)$ in $\mathbf{R} \times I$. Then $F=\cup_{n}\left\{x_{n, i}\right\}_{i=1}^{\infty}$ is sequentially closed, but not closed since $(p, q)$ is in its closure.

If $X$ is any topological space, one may impose a sequential topology on it by taking as open sets of the new topology all the sequentially open sets of the original topology. We denote this new space by $S(X)$ and call it the sequential coreflection of $X$. If $X$ was sequential, $S(X)$ will have the same topology as $X$; if $X$ was not sequential, $S(X)$ will have a strictly finer topology. For a more extensive discussion, see [D]. We will need the fact that if $X$ is a topological space and $A$ is a closed subset of $X$ which is sequential in its inherited topology, then the topology which $A$ inherits from $S(X)$ is the same one it inherits from $X$. To see this, observe that $S(X)$ has the same convergent sequences as $X$; the topology $A$ inherits from $X$ is 
sequential by hypothesis and the topology it inherits from $S(X)$ is sequential since $A$ is closed in $S(X)$. Thus both topologies are completely determined by their convergent sequences which are the same.

2. Free topological groups and $k_{\omega}$-spaces. Let $(X, p)$ be a Tychonoff space with basepoint $p$. The Graev free topological group over $(X, p)$ is a topological group $F_{G}(X, p)$ which is algebraically the free group on $X \backslash\{p\}$ and whose topology is the finest topology compatible with the group structure making the "insertion of generators" $\eta:(X, p) \rightarrow F_{G}(X, p)$ continuous $(\eta(p)$ is the group identity $e)$. $F_{G}(X, p)$ has the usual properties associated with the word "free"; in particular, any continuous pointed map $f:(X, p) \rightarrow(G, e)$ into a topological group extends uniquely to a continuous homomorphism $\hat{f}: F_{G}(X, p) \rightarrow(G, e)$.

While the topology of $F_{G}(X, p)$ can be unpleasant in general [F-O-T], [H-M], it is tractable if $X$ is a $k_{\omega}$-space. A topological space is called a $k_{\omega}$-space when it is the weak union of an increasing sequence of compact Hausdorff subspaces. The

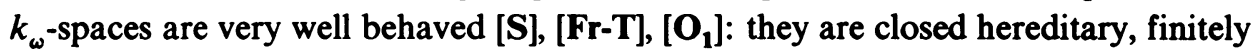
productive, preserved by countable disjoint (or pointed) unions, and preserved by Hausdorff quotients.

The spaces $S_{n}$ and $S_{\omega}$ are $k_{\omega}$-spaces.

If $(X, p)$ is a $k_{\omega}$-space, then $F_{G}(X, p)$ is topologically, (and algebraically) the weak union of the subspaces $\left(F_{G}(X, p)\right)_{n}$ consisting of words of reduced length $<n$. Further, each of the subspaces $\left(F_{G}(X, p)\right)_{n}$ is the quotient of the product $\left(X \cup_{p} X\right)^{n}$ (the cartesian product of $n$ factors, each a pointed union of two copies of $X$ ) in a natural way. For details, see $\left[\mathbf{O}_{\mathbf{1}}\right]$.

If $G$ is a topological group and $A$ is a subset of $G$, we say that $A$ generates $G$ provided that $A$ algebraically generates $G$ and that $G$ has the finest topology compatible with both the group structure and the original topology on $A$ (that is, any strictly finer group topology on $G$ would induce a strictly finer topology on the subset $A$ ). Theorem 1 of [MMO] states that if $A$ generates $G$ and $A$ is a $k_{\omega}$-space, then $G$ is also a $k_{\omega}$-space in a natural way. We will need the following related result:

LEMMA 2.1. Let $G$ be a topological group and $A$ a subset which generates it and contains the identity. Suppose $A$ is a $k_{\omega}$-space. Then the map $\hat{f}_{:} F_{G}(A, e) \rightarrow(G, e)$ which extends the inclusion $f: A \hookrightarrow G$ is a quotient map.

Proof. Write $A=\cup_{i} A_{i}$, where the basepoint is in $A_{1}$ and the $A_{i}$ 's form an increasing sequence of compact Hausdorff subspaces of $A$ which determines the topology of $A$. Then by the formulation in [MMO], the $i$ th compact Hausdorff subset of $F_{G}(A, e)$ (in a sequence determining the topology) may be taken to be the set of words which are products of at most $i$ elements of $\eta\left(A_{i}\right)$, or their inverses, and the $i$ th compact subset of $G$, in the quotient topology under $\hat{f}$, may be taken to be the set of products of at most $i$ elements of $\hat{f} \eta\left(A_{i}\right)$, or their inverses. However, this is precisely the topology that $G$ already has as a group generated by $A$, according to the proof of Theorem 1 of [MMO]. 


\section{Sequential conditions in free groups.}

THeOREM 3.1. A $k_{\omega}$-space $(X, p)$ is sequential if and only if its Graev free topological group, $F_{G}(X, p)$, is sequential.

Proof. Since $(X, p)$ can be embedded as a closed subspace of $F_{G}(X, p)$, it must be sequential if $F_{G}(X, p)$ is. On the other hand, suppose $(X, p)$ is sequential; write $X=\cup_{i} X_{i}$, where $p$ is in $X_{1}$ and the $X_{i}$ 's form an increasing sequence of compact Hausdorff subspaces of $X$ which determines the topology of $X$. Then each of the spaces $X_{i} \cup_{p} X_{i}$ is sequential and compact, so (by the theorem of Boehme) each $\left(X_{i} \cup_{p} X_{i}\right)^{2}$, and by induction each $\left(X_{i} \cup_{p} X_{i}\right)^{n}$, is sequential. Thus the weak union (in fact, $k_{\omega}$-decomposition) $\left(X \cup_{p} X\right)^{n}=\cup_{i}\left(X_{i} \cup_{p} X_{i}\right)^{n}$ is sequential for each $n$. It follows that the quotients $\left(F_{G}(X, p)\right)_{n}$ are sequential, and so the weak union $F_{G}(X, p)$ must be also.

COROLLARY 3.2. Let $G$ be a topological group generated (in our special sense) by a subset $A$. If $A$ is a sequential $k_{\omega}$-space, then so is $G$.

Proof. $G$ is a $k_{\omega}$-space by [MMO]. It is sequential since by Lemma 2.1 , it is a quotient of the sequential space $F_{G}(A, p)$.

The free product $G * H$ of two topological groups $\left[\mathbf{G r}_{2}\right],\left[\mathbf{O}_{2}\right]$ is generated by $G \cup_{e} H$. Hence:

COROLlaRY 3.3. Let $G$ and $H$ be topological groups which are sequential $k_{\omega}$-spaces. Then their free product $G * H$ is also a sequential $k_{\omega}$-space.

From Theorem 3.1 we see that there are free topological groups which are sequential. Naturally, one wonders what happens when the " $k_{\omega}$ " requirement is dropped. As before, if the Graev free topological group $F_{G}(X, p)$ is sequential, then $(X, p)$ must be sequential. The following example shows that the implication in the other direction fails:

EXAmple 3.4. Let $X$ be the pointed union of $(2, p)$ with $(W, q)$. Since 2 and $W$ are sequential, so is $X$. But by [F-O-T], $F_{G}(X, p)$ contains a closed subspace homeomorphic to $2 \times W$; since $2 \times W$ is not sequential, neither is $F_{G}(X, p)$.

In Theorem 6.6 of [D], Dudley proves the following: let $P$ denote the set of all real functions of a real variable, with the topology of pointwise convergence. Let $S(P)$ denote the sequential coreflection of $P . P$ is of course a topological group; however, assuming the continuum hypothesis, $S(P)$ is not a topological group: addition of functions is discontinuous. If we use the space $F_{G}(X, p)$ of the above example, we do not need the continuum hypothesis to obtain an example of a topological group whose sequential coreflection is not a compatible topology:

EXAMPLE 3.5. Let $X$ be as in Example 3.4. $F_{G}(X, p)$ is a topological group. Since it is not sequential, its sequential coreflection $S\left(F_{G}(X, p)\right)$ has a strictly finer topology, which, however, induces the original topology on the closed sequential subset $\eta(X)$. However, the free topology on $F_{G}(X, p)$ is the finest such topology compatible with the group operation; hence, the group operation is discontinuous in the topology of $S\left(F_{G}(X, p)\right)$. 
We now turn to the problem of determining the sequential order of a free topological group.

LEMMA 3.6. Let $(X, p)$ be a Tychonoff space and suppose there is a sequence of distinct terms $x_{1}, x_{2}, x_{3}, \ldots$ in $X$ converging to $p$. Let $T$ be the set $\left\{p, x_{1}, x_{2}, \ldots\right\}$. Then $F_{G}(T, p)$ is contained in $F_{G}(X, p)$ as a closed subgroup.

Proof. For a similar result, see Proposition 5.3 of $\left[\mathbf{O}_{1}\right]$. We give an argument using a method of proof developed in [H-M-T]. Let $\beta X$ be the Stone-Čech compactification of $X$. The inclusion $T \subset X \subset \beta X$ is an inclusion of the compact set $T$ in the compact space $\beta X$ and yields continuous homomorphisms $F_{G}(T, p) \rightarrow$ $F_{G}(X, p) \rightarrow F_{G}(\beta X, p)$ where it may be easily checked (since $F_{G}(\beta X, p)$ is a $k_{\omega}$-space) that $F_{G}(T, p) \rightarrow F_{G}(\beta X, p)$ is a closed embedding. Hence the image of $F_{G}(T, p)$ is closed in $F_{G}(X, p)$.

THEOREM 3.7. Let $(X, p)$ be a Tychonoff space and suppose there is a sequence of distinct terms $x_{1}, x_{2}, x_{3}, \ldots$ in $X$ converging to $p$. Then there is a closed embedding $f:\left(S_{\omega}, s_{0}\right) \rightarrow F_{G}(X, p)$.

Proof. In view of Lemma 3.6, it will suffice to produce a closed embedding $f$ : $\left(S_{\omega}, s_{0}\right) \rightarrow F_{G}(T, p)$. Enumerate the sequences of $S_{\omega}$ as follows: denote the single sequence of $S_{1}$ by $t_{1}=s_{1}, s_{2}, s_{3}, \ldots$ Denote the sequence of $S_{2}$ converging to $s_{1}$ by $t_{2}=s_{1,1}, s_{1,2}, s_{1,3}, \ldots$ Use a diagonalization process to enumerate all the sequences of $S_{\omega}$; the limits of the sequences $t_{3}, t_{4}, t_{5}, t_{6}, t_{7}, t_{8}, \ldots$ are respectively $s_{1,1}, s_{2}, s_{1,1,1}, s_{1,2}, s_{2,1} s_{3}, \ldots$ The basic idea in constructing the function $f$ is to map each sequence $t_{i}$ into the set of words of $F_{G}(T, p)$ which have reduced length precisely $i$. Let $f\left(s_{0}\right)=p$. Let $f\left(t_{1}\right)=T \backslash\{p\}$, with $f\left(s_{n}\right)=x_{n}$. Let $f\left(t_{2}\right)=$ $x_{1} x_{1}, x_{1} x_{2}, x_{1} x_{3}, \ldots ;$ let $f\left(t_{3}\right)=x_{1} x_{1} x_{1}, x_{1} x_{1} x_{2}, x_{1} x_{1} x_{3}, \ldots ;$ and let

$$
f\left(t_{4}\right)=x_{2} x_{1} x_{1} x_{1}, x_{2} x_{2} x_{2} x_{2}, x_{2} x_{3} x_{3} x_{3}, \ldots
$$

Inductively, if the sequence $t_{i}$ converges to $s_{j, k, \ldots, m}$, then $f\left(t_{i}\right)=f\left(s_{j, k, \ldots, m}\right) x_{1}^{r}$, $f\left(s_{j, k, \ldots, m}\right) x_{2}^{r}, \ldots$, where $r=i-(j+k+\ldots+m)$. Note that in view of the way the $s_{j, k}, \ldots$ were enumerated and the way the $i$ are defined, the exponents $r$ are always positive, and the function $f$ is clearly one-to-one. Since $f$ was chosen to preserve sequential convergence and $S_{\omega}$ is sequential, $f$ is continuous. That $f$ is a closed embedding follows readily from the fact that the intersection of its image with each $\left(F_{G}(T, p)\right)_{n}$ consists of precisely $n$ convergent sequences with their limits.

COROLlARY 3.8. Let $(X, p)$ be a Tychonoff space and suppose it contains some point which is the limit of a nonconstant sequence. Then $F_{G}(X, p)$ contains a closed subspace homeomorphic to $\left(S_{\omega}, s_{0}\right)$.

Proof. A convergent subsequence of distinct terms may be extracted from the given sequence in $X$. If the limit point is $p$, Theorem 3.7 applies. But by [Gr $\mathbf{~}]$, $F_{G}(X, p)$ is (up to isomorphism of topological groups) independent of the choice of basepoint $p$ in $X$.

Since $\left(S_{\omega}, s_{0}\right)$ is sequential of order $\omega_{1}$, we obtain: 
THEOREM 3.9. Let $(X, p)$ be any Tychonoff space, and suppose $F_{G}(X, p)$ is sequential but not discrete. Then it is sequential of order $\omega_{1}$.

Proof. As was noted before Example 3.4, $X$ is sequential, and $X$ is not discrete (or $F_{G}(X, p)$ would be also). Hence $X$ contains a nonconstant convergent sequence, and $F_{G}(X, p)$ contains a copy of $\left(S_{\omega}, s_{0}\right)$. Hence the sequential order of $F_{G}(X, p)$ is at least $\omega_{1}$; since that is the maximum possible sequential order of any space, it must be exactly $\omega_{1}$.

It would be nice to take Corollary 3.8 one step further, leading to the following:

Question 3.10. Let $F_{G}(X, p)$ contain some nontrivial convergent sequence. Must it contain a copy of $\left(S_{\omega}, s_{0}\right)$ ? A closed copy?

An affirmative answer would follow from an affirmative answer to:

Question 3.11. Let $F_{G}(X, p)$ contain a nontrivial convergent sequence. Need $(X, p)$ contain a nontrivial convergent sequence?

4. Nongroupability of $S_{\omega}$. Since we have now embedded $\left(S_{\omega}, s_{0}\right)$ as a closed subspace of $F_{G}(X, p)$ for many spaces $(X, p)$, it is natural to ask if it can be embedded as a subgroup. Clearly, the embedding given here does not make it a subgroup, since it includes all elements of $\eta(T) \subset F_{G}(T, p)$ but not all products of three such elements. In fact, no embedding can make it a subgroup. The following result is even stronger:

THEOREM 4.1. There is no topological group homeomorphic to $\left(S_{\omega}, s_{0}\right)$.

Loosely, our strategy will be to show that while diagonals generally fail to converge in $S_{\omega}$, in a topological group diagonals generally do converge. We need this lemma:

LEMMA 4.2. In $S_{\omega}$ let $y_{0}$ be a fixed but arbitrary point. Let $y_{1}, y_{2}, y_{3}, \ldots$ be a sequence converging to $y_{0}$. For each $i$ let $y_{i, 1}, y_{i, 2}, y_{i, 3}, \ldots$ be a sequence converging to $y_{i}$. Suppose all the points $y_{0}, y_{i}, y_{i, j}$ are distinct. Then there is a function $f: \mathbf{N} \rightarrow \mathbf{N}(\mathbf{N}$ is the natural numbers) with $f(i) \geqslant i$ for all $i$, such that the sequence $y_{i, f(i)}, i=$ $1,2,3, \ldots$ fails to converge.

Proof. We must describe the topology of $S_{\omega}$ in more detail. Consider the labelling of sequences in $S_{\omega}$ given in the proof of Theorem 3.7; let $T_{n}$ denote the sequence $t_{n}$ and its limit. Instead of considering $S_{\omega}$ to be the weak union of the spaces $S_{n}$, we may regard it as a quotient of the disjoint union of the sequences $T_{n}$. With this viewpoint, we see that a subset $A$ of $S_{\omega}$ is closed if and only if every intersection $A \cap T_{n}$ is closed. If $n \neq m, T_{n}$ and $T_{m}$ intersect in at most one point, and if there is such a point it is the (unique) limit point of exactly one of $T_{n}$ or $T_{m}$. Each point of $S_{\omega}$ is the limit point of exactly one $T_{n}$ and, except for $s_{0}$, a nonlimit point of exactly one other. A sequence converges only if it is eventually in some $T_{n}$, and then, if it is not eventually constant, it converges to the limit point of that $T_{n}$.

Now, $y_{0}$ is the limit point of some $T_{n}$, say $T_{n_{0}}$, and the sequence $y_{1}, y_{2}, y_{3}, \ldots$ is eventually in $T_{n_{0}}$. Each $y_{i}$ is the limit point of some $T_{n}$, say $T_{n_{i}}$, and the sequence $y_{i, 1}, y_{i, 2}, y_{i, 3}, \ldots$ is eventually in $T_{n_{i}}$. Pick $f$ so that $f(i)>i$ and $y_{i, f(i)} \in T_{n_{i}}$ for each $i$. 
Now $y_{i, f(i)}$ is a nonlimit point of $T_{n_{i}}$ for each $i$; hence at most two $y_{i, f(i)}$ lie in any $T_{n}$, the sequence $y_{i, f(i)}, i=1,2,3, \ldots$, is not eventually in any $T_{n}$, and so this sequence cannot converge.

Proof of Theorem 4.1. Suppose $S_{\omega}$ to be a topological group. Let $x_{0}$ be a point in $S_{\omega}$ and let $x_{1}, x_{2}, x_{3}, \ldots$ be a sequence converging to $x_{0}$ such that all the points $x_{0}, x_{1}, x_{2}, \ldots$ are distinct and none is the group identity. Denote the multiplication on $S_{\omega}$ by $m($, ).

We shall select some points in $S_{\omega}$ to fill the roles of the points in the statement of Lemma 4.2. Let $y_{0}$ be $m\left(x_{0}, x_{0}\right)$. Let $y_{i}$ be $m\left(x_{0}, x_{i}\right)$ for $i=1,2,3, \ldots$ Then $y_{0}$ and the $y_{i}$ are distinct and $\lim y_{i}=y_{0}$. We next choose the $y_{1, j}, j=1,2,3, \ldots$ as follows: the sequence $m\left(x_{j}, x_{1}\right), j=1,2,3, \ldots$, converges to $m\left(x_{0}, x_{1}\right)$. It is eventually disjoint from the sequence $m\left(x_{0}, x_{i}\right)$ because the two sequences have different limits. Hence there is a positive integer $k_{1}$ such that for $j>k_{1}+1$, the points $m\left(x_{j}, x_{1}\right)$ are distinct from all points chosen thus far. Pick as $y_{1, j}$ the point $m\left(x_{k_{1}+j}, x_{1}\right)$. Having picked sequences $y_{i, j}, j=1,2,3, \ldots$, for $i<n$, proceed by induction: Note that $m\left(x_{j}, x_{n}\right), j=1,2,3, \ldots$, converges to $m\left(x_{0}, x_{n}\right)$ and is eventually disjoint (for $j>k_{n}$ ) from the set consisting of $y_{0}$, the $y_{i}$ 's, and all the previously chosen $y_{i, j}$ 's; pick $k_{n}$ sufficiently large and let $y_{n j}=m\left(x_{k_{n}+j}, x_{n}\right)$.

The $y_{0}, y_{i}$ 's, and $y_{i, j}$ 's we have selected from $S_{\omega}$ meet the conditions of Lemma 4.2. Now let $f: \mathbf{N} \rightarrow \mathbf{N}$ be any function with $f(i) \geq i$ for all $i$. $\lim x_{i}=x_{0}$ (all limits are taken as $i \rightarrow \infty)$; also $\lim x_{f(i)+k_{i}}=x_{0}$ since if $i \geqslant M, f(i)+k_{i} \geqslant M$. Hence in $S_{\omega} \times S_{\omega}, \lim \left(x_{f(i)+k_{i}}, x_{i}\right)=\left(x_{0}, x_{0}\right)$. Now by continuity of multiplication in $S_{\omega}$,

$$
\lim y_{i, f(i)}=\lim m\left(x_{f(i)+k_{i}}, x_{i}\right)=m\left(x_{0}, x_{0}\right)=y_{0}
$$

contradicting Lemma 4.2 and completing the proof of Theorem 4.1.

\section{REFERENCES}

[A-Fr] A. V. Arhangel'skii and S. P. Franklin, Ordinal invariants for topological spaces, Michigan Math. J. 15 (1968), 313-320.

[B] T. K. Boehme, Linear s-spaces, (Proc. Sympos. Convergence Structures, Univ. of Oklahoma, Norman, 1965), Univ. of Oklahoma, Norman, 1965.

[D] R. M. Dudley, On sequential convergence, Trans. Amer. Math. Soc. 112 (1964), 483-507; Corrections, ibid. 148 (1970), 623-624.

[F-O-T] T. H. Fay, E. T. Ordman and B. V. S. Thomas, The free topological group over the rationals, General Topology and Appl. 10 (1979), 33-47.

[Fr, ] S. P. Franklin, Spaces in which sequences suffice. I, Fund. Math. 57 (1965), 107-115.

$\left[\mathrm{Fr}_{2}\right] \ldots$ Spaces in which sequences suffice. II, Fund. Math. 61 (1967), 51-56.

[Fr-T] S. P. Franklin and B. V. S. Thomas, $A$ survey of $k_{\omega}$-spaces, (Topology Proceedings, vol. 2, no. 1, Proc. 1977 Topology Conf., Baton Rouge, La.), Auburn Univ., Auburn, Ala., 1978, pp. 111-124.

[Gr $\mathbf{G}_{1}$ ] M. I. Graev, Free topological groups, Izv. Akad. Nauk SSSR Ser. Mat. 9 (1945), 3-64; English transl., Amer. Math. Soc. Transl. (1) 8 (1962), 305-364.

$\left[\mathbf{G r}_{2}\right] \_$, On free products of topological groups, Izv. Akad. Nauk SSSR Ser. Mat. 14 (1950), 343-354. (Russian)

[H-M] David C. Hunt and Sidney A. Morris, Free subgroups of free topological groups, (Proc. Second Internat. Conf. Theory of Groups, Canberra, 1973), Lecture Notes in Math., vol. 372, Springer-Verlag, Berlin and New York, 1974, pp. 377-387.

[H-M-T] J. P. L. Hardy, Sidney A. Morris and H. B. Thompson, Applications of the Stone- $\grave{C}$ ech compactification to free topological groups, Proc. Amer. Math. Soc. 55 (1976), 160-164.

[M-M-O] John Mack, Sidney A. Morris and Edward T. Ordman, Free topological groups and the projective dimension of a local compact abelian group, Proc. Amer. Math. Soc. 40 (1973), 303-308. 
[0, Edward T. Ordman, Free $k$-groups and free topological groups, General Topology and Appl. 5 (1975), 205-219.

$\left[\mathrm{O}_{2}\right] \longrightarrow$, Free products of topological groups which are $k_{\omega}$-spaces, Trans. Amer. Math. Soc. 191 (1974), 61-73.

[R] M. Rajagopalan, Sequential order and spaces $S_{n}$, Proc. Amer. Math. Soc. 54 (1976), 433-438.

[S] N. E. Steenrod, A convenient category of topological spaces, Michigan Math. J. 14 (1967), 133-152.

Department of Mathematics, New England College, Hennikgr, New Hampshime 03242 (Current address of E. T. Ordman)

Department of Mathematical Sciences, Memphis State University, Memphis, Tennesseb 38152

Current address (B. V. Smith-Thomas): Department of Mathematics, University of Alabama, Birmingham, Alabama 35294 